\title{
Il costo sociale del morbillo in età pediatrica. L'epidemia a Palermo nel 1996-97*
}

\author{
C. Lucioni, ${ }^{1}$ S. Ciriminna, ${ }^{2}$ P. Di Carlo, ${ }^{3}$ S. Mazzi, ${ }^{1}$ G. Serra e L. Titone \\ Istituto di Economia Sanitaria, Milano \\ Ispettorato Sanitario Regione Sicilia, Palermo \\ 3 Divisione Malattie Infettive, Ospedali Riuniti, Reggio Calabria \\ 4 Unità di Farmacoeconomia, SmithKline Beecham Italia \\ 5 Istituto Malattie Infettive, Università degli Studi, Palermo
}

\section{Summary}

\begin{abstract}
Objective
To determine the direct and indirect costs associated with a measles epidemic occurring between September 1996 and August 1997 in Palermo (Italy) in paediatric-aged patients. Design

A total of 2,029 cases of measles in a paediatric patient population were identified from a total of 38 paediatricians databases ( $24 \%$ of total). An extrapolation to the general population was then performed to estimate a total of 9,059 cases. Patient information obtained from the database such as patient age, risk factors, complications, vaccination history, as well as caretaker's profession were included in a questionnaire compiled for each patient.
\end{abstract}

Setting

Inpatient and outpatient clinics in Palermo, Italy.

Patients and participants

Participants were paediatric-aged patients who had been diagnosed with measles. Included in the study was a group of previously vaccinated patients (6\%).

\section{Main outcome measures and results}

The average cost of care was 464.000 Italian lire (Lit.) per case with a total cost of Lit. 4,2 billion for the entire epidemic. The direct costs comprehended $46.6 \%$ (Lit. 217.000 per case) of the total costs related to the measles epidemic and were subdivided according to in-patient care (55.4\%), paediatric outpatient visits $(33.5 \%)$ and drugs $(9.7 \%)$. The average health-care cost associated to previously vaccinated patients $(6 \%)$ was lower than for non-vaccinated patients, Lit. 110.000 vs Lit. 223.000 per case, respectively.

\section{Conclusion}

The demographic and economic data obtained highlights not only the social and economic impact of the epidemic, but also provides relevant information useful for cost-effectiveness analysis.

\footnotetext{
* I peer reviewer per questo articolo sono stati coordinati da G. Fattore.
} 


\section{Introduzione}

Le conseguenze delle malattie infettive costituiscono una minaccia costante e un onere economico per la società, qualunque sia il suo grado di sviluppo, tanto che nell'obiettivo di controllarle molti Paesi hanno deciso di investire parti consistenti del proprio bilancio sanitario. ${ }^{[1]}$

Nel corso degli ultimi cinquant'anni sono stati compiuti grandi passi avanti nel trattamento di un gran numero di agenti infettivi, grazie all'impiego di diversi principi attivi. Ma solo con l'immunizzazione si è stati in grado di prevenire la morbilità e la mortalità associate ad alcune importanti malattie infettive. Per avere un ordine di grandezza indicativo a livello mondiale, basti pensare che fino a venti anni fa morivano ogni anno di morbillo, tetano, pertosse, difterite, tubercolosi o poliomielite circa cinque milioni di bambini, e altri milioni rimanevano disabili permanenti. Senza l'Expanded Programme on Immunization (EPI), lanciato nel 1974 dalla World Health Organization (WHO), si stima che ci sarebbero circa 3,2 milioni di decessi ogni anno, dovuti a morbillo, pertosse e tetano neonatale; tuttavia, malgrado l'EPI, queste tre malattie continuano a provocare ogni anno 1,7 milioni di morti. ${ }^{[2]}$

Il morbillo è stato a lungo materia favorita di studio tra gli epidemiologi. Sull'incidenza di questa malattia esiste da tempo un' ampia documentazione, che mette in luce la ricorrenza delle epidemie che si verificano persino ogni due anni anche in condizioni endemiche quali quelle delle grandi comunità dell'Europa e del Nord America; ${ }^{[3]}$ tanto che già più di un secolo $\mathrm{fa}^{[4]}$ era stata sollevata la questione delle possibili origini di tale modello ciclico. ${ }^{[5]}$

Tralasciando queste curiosità aneddotiche, occorre fare attenzione a un persistente, diffuso malinteso a proposito del morbillo, cioè che si tratti di una malattia infantile praticamente senza conseguenze. ${ }^{[6]}$ In realtà, si tratta invece di una malattia che può diventare grave, poiché di fatto comporta frequenti complicanze, dall'infezione dell'apparato auricolare medio alla broncopolmonite. ${ }^{[1]}$

Negli Stati Uniti, nel decennio precedente all'introduzione del vaccino virale vivo attenuato (1963) venivano notificati ogni anno ai Centers for Disease
Control (CDC) fra i 300.000 e gli 800.000 casi di morbillo, ${ }^{[7]}$ che comportavano polmonite nel $2,7 \%$ $6,5 \%$ dei casi ed encefalite nello $0,1 \%$. Il ricovero ospedaliero era necessario nel 10,8\%-21\% di tutti i pazienti. ${ }^{[8,9]}$

Con la diffusione del vaccino, l'incidenza è scesa a 75.000 casi all'anno, fino a un minimo di 1.497 casi nel 1983. Tuttavia, alla fine degli anni '80 si è assistito a una preoccupante ripresa, contrassegnata da epidemie nonché da aumento dell'incidenza nei giovani adulti: nella maggior parte dei casi la causa appare essere l'incompleta immunizzazione delle coorti infantili (mancato raggiungimento dei livelli protettivi di anticorpi o decadimento dei livelli protettivi nel tempo). ${ }^{[7-9]}$

Analogamente, si può apprezzare la scala degli effetti devastanti che il morbillo comporta sulla salute infantile pensando che in Inghilterra e Galles, nel 1967 (l'anno prima che prendesse avvio la fase di immunizzazione di massa), erano stati notificati 460.407 casi. ${ }^{[10]}$ Ogni 1.000 notifiche, si verificano 0,2 decessi, 10 ricoveri, 10 complicanze neurologiche e 40 respiratorie. Anche nei casi lievi non ospedalizzati si osserva un notevole stato di deprivazione sia per il bambino che per la famiglia. ${ }^{[1]}$

In Belgio, nel programma di sorveglianza del morbillo, sono state riportate complicanze nel $15 \%$ dei casi tra il 1982 e il $1990 .^{[12]} \mathrm{Si}$ verifica approssimativamente un caso di encefalite ogni 2.000 casi notificati; i sopravvissuti subiscono spesso un danno permanente al cervello e un ritardo mentale. ${ }^{[13]}$

Uno studio ${ }^{[14]}$ ha stimato che in Francia, senza l'adozione del triplo vaccino (morbillo, parotite, rosolia) nel corso di 25 anni si sarebbero verificati per quanto riguarda specificamente il morbillo 9 milioni di casi, 2.000 encefaliti, 900 complicanze e 500 decessi.

In Italia, la vaccinazione contro il morbillo non è obbligatoria. Per farsi un'idea quantitativa dell'attuale consistenza della malattia può essere di aiuto la tabella I, dove sono riportate alcune serie di dati relativi alle notifiche dei casi di morbillo presentate rispettivamente in Italia, in Sicilia, a Palermo e provincia negli anni ' 90 .

I limiti che ai dati delle notifiche di malattie infettive in Italia derivano dal fenomeno della sottonotifica sono noti; tuttavia, ipotizzando di tale fenomeno una 
Tabella I. Notifiche di morbillo in Italia, in Sicilia e a Palermo dal 1991 al 1997

\begin{tabular}{|c|c|c|c|c|c|c|c|}
\hline Anno & 1991 & 1992 & 1993 & 1994 & 1995 & 1996 & 1997 \\
\hline Italia & 24.184 & 64.784 & $\begin{array}{c}16.283 \\
\text { di cui: fino a } 14 \text { anni } \\
\text { oltre i } 14 \text { anni }\end{array}$ & $\begin{array}{l}5.945 \\
48 \% \\
52 \%\end{array}$ & $\begin{array}{r}37.036 \\
\text { incidenza }\end{array}$ & $\begin{array}{r}28.976 \\
0,5 \%\end{array}$ & \\
\hline Sicilia & 749 & 8.797 & 157 & 53 & 589 & 4.979 & 5.600 \\
\hline Palermo & 645 & 3.250 & 19 & 9 & di cui: & $\begin{array}{l}290 \\
\text { : fino a } 14 \text { anni } \\
\text { oltre } 14 \text { anni }\end{array}$ & $\begin{array}{r}3.473 \\
96 \% \\
4 \%\end{array}$ \\
\hline
\end{tabular}

Fonte: Annuari Statistici ISTAT (per i dati relativi all Italia) e dati della Regione Sicilia ${ }^{[23]}$

certa uniformità di distribuzione nel tempo, la tabella può essere indicativamente utilizzata.

In particolare, essa indica che nel 1996 il quoziente medio di notifica per tutto il Paese è stato di 50,5 per 100.000 abitanti (corrispondente a un tasso di incidenza dello $0,5 \%$, ovvero di un caso ogni 2.000 abitanti). Sempre in tutto il Paese, nel 1994 il morbillo era distribuito approssimativamente per metà tra la popolazione in età pediatrica $(48 \%$ dei casi nei soggetti fino a 14 anni di età) e per l'altra metà in quella adulta (molto diversamente, come si può vedere, da quanto è stato notificato a Palermo e provincia nel 1997, dove il morbillo si è manifestato quasi unicamente cioè nel $96 \%$ dei casi nella popolazione pediatrica).

Si può vedere che gli andamenti nel tempo delle tre serie sono discretamente correlati tra loro, per quanto si debba dare per scontata una certa disomogeneità territoriale dell'epidemiologia. Le ricorrenze epidemiche appaiono abbastanza delineate, con una ciclicità di circa quattro anni. Peraltro, in nessuna serie è possibile verificare l'esistenza di una tendenza temporale, per cui non si può affermare su questa base se $\mathrm{i}$ casi sono in crescita o in diminuzione.

\section{Un recente caso di epidemia di morbillo in Italia}

In una rassegna, per quanto non esaustiva, della recente letteratura farmacoeconomica sul morbillo non si trovano lavori sul costo sociale della malattia come tale. Infatti, tutta la ricerca è ormai rivolta alla valutazione economica della vaccinazione preventiva di massa ${ }^{[10]}$ eventualmente secondo parti- colari strategie ${ }^{[15]}$ o dello screening, ${ }^{[16]}$ o della rivaccinazione selettiva in caso di epidemie. ${ }^{[17,18]}$

Il case study oggetto del presente lavoro vuole essere un contributo alla stima del costo sociale del morbillo in Italia, stima quindi precedente alla valutazione economica delle diverse strategie di contrasto. In linea generale, gli studi sui costi sociali delle malattie promuovono la raccolta di una serie di dati di base che possono poi essere utilizzati in analisi economiche più avanzate, fondate oltre che sui dati di costo della strategia sanitaria da valutare sulla buona qualità dei dati relativi all'efficacia delle risorse consumate. Quest'ultimo genere di informazione è proprio quello che uno studio sul costo sociale della malattia concorre a costruire, rappresentando quindi un'apprezzabile linea di partenza per l'eventuale successiva valutazione delle opportunità di intervento.

Nello specifico, lo studio si propone di analizzare alcuni risvolti economici di una recente epidemia di morbillo verificatasi a Palermo e provincia a cavallo tra il 1996 e il 1997, in proporzioni quanto meno paragonabili a quelle rilevate nella precedente epidemia del 1991-92.

Si possono percepire le reali dimensioni del fenomeno se si considera che tra il mese di settembre 1996 e quello di giugno 1997 si sono presentati al Pronto Soccorso del Presidio Ospedaliero G. Di Cristina (il più grosso polo pediatrico del capoluogo) 1.642 pazienti affetti da morbillo (in età media di 56 anni), dei quali 558 sono stati ricoverati nello stesso Ospedale, presso l'Istituto di Malattie Infettive dell'Università di Palermo. Di questi ultimi, il $72 \%$ presentava delle complicanze. ${ }^{[19]}$ 


\section{Materiali e metodi}

Il presente lavoro è una ricerca di carattere empirico, basata cioè su dati appositamente rilevati che riguardano le principali risorse impegnate nell'epidemia di morbillo verificatasi a Palermo nel 1996-97.

Per la rilevazione dei dati è stato messo a punto un questionario in forma di scheda, somministrato nel corso di interviste rivolte a una parte dei pediatri di base che esercitano a Palermo. Su ogni questionario uno per ciascun caso di morbillo sono state così raccolte, utilizzando i database professionali (di tipo elettronico o cartaceo) dei pediatri intervistati, alcune informazioni (tutte in forma rigorosamente anonima) sia sul pediatra (USL e quartiere di localizzazione, numero di assistiti, casi di morbillo) sia sul singolo caso di malattia: ad esempio, le risorse impegnate sul piano sanitario (farmaci, ricoveri), nonché alcune informazioni di tipo clinico-epidemiologico (età del paziente, complicanze, fattori di rischio) e socio-economico (stato occupazionale della madre del paziente). Sono stati presi in considerazione tutti i casi di malattia verificatisi dal settembre 1996 all'agosto 1997. A rilevazioni terminate (per un ammontare di 2.029 schede-questionario), tutte le informazioni raccolte sono state trasferite su un database Excel.

Sono stati coinvolti, su base volontaria, 38 dei 159 pediatri convenzionati esercitanti a Palermo vale a dire poco meno di un quarto $(23,9 \%)$ del numero complessivo. Il numero dei loro assistiti (31.565), cioè la popolazione campionata, corrisponde a circa il $22,4 \%$ dell'intera popolazione pediatrica residente a Palermo (141.077 soggetti); analizzando tale quota campionaria a livello delle cinque USL della città esistenti nel 1996-97 si ha la distribuzione territoriale mostrata nella tabella II.

Nel complesso, dunque, pur non trattandosi di un campione formato su base rigorosamente casuale, e neppure stratificato con criterio davvero uniforme, la sua elevata numerosità unita all'assenza di evidenti distorsioni lo rende accettabilmente rappresentativo in rapporto alle finalità conoscitive che la ricerca si propone. Sulla base di queste considerazioni, in particolare, è parso corretto utilizzare la quota campionaria generale $(22,4 \%)$ per ricavare dal suo reciproco il fattore di proiezione con cui espandere i risultati dalla scala di grandezza del campione a quella della popolazione: applicando questo fattore ai casi di morbillo rilevati nel campione sono infatti stati stimati 9.059 casi di morbillo a livello di tutta la popolazione pediatrica di Palermo città.

Si tratta comunque di una base empirica circoscritta alla popolazione in età pediatrica, residente a Palermo. Della popolazione colpita in età adulta questa ricerca non può dire nulla.

In ogni valutazione economica è cruciale la definizione del punto di vista dell' analisi; nel presente lavoro, trattandosi di uno studio sul costo sociale della malattia, il punto di vista dell'analisi è quello della società nel suo complesso.

In generale, le due componenti misurabili (sia pure con diversi gradi di difficoltà) del costo sociale di una malattia sono i costi diretti e quelli indiretti, cui essa dà luogo. I primi sono costituiti soprattutto da quelli a carico del Servizio Sanitario Nazionale per la prevenzione, la diagnosi, il trattamento della malattia stessa; essendo legati a prestazioni effettivamente erogate, alle quali corrispondono prezzi o tariffe ben precise, sono quelli relativamente più facili da stimare. I costi indiretti (cioè il valore economico del tempo perso a causa della malattia) sono innanzitutto quelli dovuti alle perdite di produzione del sistema economico causate dalla morbilità e dalla mortalità nei soggetti attivi colpiti; poiché non vi corrispondono esborsi monetari veri e propri, è necessario ricorrere ad alcune stime

ad esempio del costo di quel lavoro che non è stato realizzato.

Tabella II. Distribuzione territoriale della quota campionaria (Palermo)

\begin{tabular}{llll}
\hline USL & $\begin{array}{l}\text { Popolazione } \\
\text { pediatrica }\end{array}$ & $\begin{array}{l}\text { Popolazione } \\
\text { campionata }\end{array}$ & $\begin{array}{l}\text { Quota } \\
\text { campionaria }\end{array}$ \\
\hline 58 & 16.177 & 5.910 & $36,5 \%$ \\
59 & 26.210 & 5.730 & $21,9 \%$ \\
60 & 32.292 & 5.640 & $17,5 \%$ \\
61 & 31.414 & 7.430 & $23,7 \%$ \\
62 & 34.984 & 6.855 & $19,6 \%$ \\
Totale & $\mathbf{1 4 1 . 0 7 7}$ & $\mathbf{3 1 . 5 6 5}$ & $\mathbf{2 2 , 4 \%}$ \\
\hline
\end{tabular}

Fonte: Dati sulla popolazione pediatrica comunicati dall Università di Palermo 
Nel presente studio sono state calcolate quattro voci di costi diretti e due di costi indiretti.

\section{I costi diretti}

\section{La spesa farmaceutica}

Per ciascun caso di morbillo, attraverso il questionario è stato rilevato il consumo extraospedaliero (ovvero in regime ambulatoriale) di farmaci, specificati col nome del rispettivo principio attivo; per ogni farmaco veniva inoltre riportato il numero di giorni durante i quali era stato somministrato al paziente (durata della terapia).

Conseguentemente, per i principi attivi menzionati nei questionari si è provveduto a individuare le relative specialità, nelle forme e confezioni usate in pediatria, nonché le dosi giornaliere, definite secondo le diverse fasce di età dei pazienti. Nel calcolare il valore della spesa farmaceutica si è adottato il prezzo pieno, cioè il prezzo al pubblico (ripreso dall' aggiornamento a novembre 1997 dell' Informatore Farmaceutico), che è il dato significativo quando il costo viene valutato secondo il punto di vista della società.

Per ciascun paziente, si è calcolato il costo del consumo giornaliero del prodotto stesso sulla base della dose giornaliera specifica per la sua età e del costo del prodotto (riportato dalla confezione all'unità posologica).

Il costo di tutta la terapia, infine, in linea generale può essere calcolato adottando diversi criteri. Ad esempio, si può ipotizzare un consumo totale di prodotto direttamente proporzionale alla durata della terapia, e quindi il costo totale si ottiene moltiplicando il costo giornaliero per il numero di giorni della stessa; tale risultato si potrebbe indicare col termine di $\bullet$ costo lineare, per sottolineare la proporzionalità continua, lineare, che corre tra la durata della terapia e il costo totale della stessa. Un secondo criterio probabilmente più realistico, soprattutto in caso di formulazioni quali sciroppi e sospensioni ipotizza invece che la confezione del prodotto, una volta aperta, anche se non completamente utilizzata per la terapia, non verrebbe comunque più riutilizzata, per il contenuto residuo, in tempi successivi; di conseguenza, il costo di tutta la tera- pia diventa il costo di una confezione anche se questa è superiore alla necessità: diventa il costo di due confezioni se una non basta, ecc.; per contrapposizione al primo, si potrebbe chiamare tale risultato - costo a gradini . È chiaro che anche con questo secondo criterio di calcolo esiste in linea di principio una proporzionalità tra la durata della terapia e il suo costo; tale proporzionalità è però discontinua, il costo cresce non in progressione lineare ma per salti, corrispondenti al costo unitario delle confezioni via via utilizzate. Nel presente lavoro si è adottato il secondo dei due criteri qui sopra proposti, ritenuto più aderente alla realtà.

Una volta ricostruiti i costi di tutte le terapie farmacologiche per tutti i pazienti, si è proceduto a sintetizzare questa miriade di informazioni calcolando i costi medi, dopo aver suddiviso i farmaci utilizzati dai pazienti in quattro grandi categorie: antipiretici e FANS; antibiotici orali; antibiotici iniettabili; altri farmaci (mucolitici, antitosse, vitamine, ecc.). Per ciascuna di queste categorie si sarebbero potuti calcolare, in linea di massima, due tipi di medie di costo: in uno suddividendo il costo complessivo dei farmaci consumati su tutti i casi di morbillo, nell'altro solo sui pazienti che avevano effettivamente consumato i farmaci. La differenza tra i due tipi di medie dovrebbe essere chiara: il primo è quello proprio di un'ottica di costo sociale, poiché indica che, al verificarsi di un'epidemia di morbillo, il costo pro capite in antipiretici o in antibiotici, ecc. è nel complesso di un certo importo, a prescindere dal fatto che alcuni pazienti assumano i farmaci e altri no. Il secondo tipo è invece più vicino a un punto di vista individuale perché indica quanto costano mediamente i farmaci in rapporto al malato di morbillo che li assume. Naturalmente, i due costi medi coincidono a livello complessivo cioè se moltiplicati per il rispettivo numero di casi. Nei risultati del presente lavoro è però stato riportato solo il primo tipo di costo medio (cioè quello ottenuto suddividendo il costo complessivo sulla generalità dei casi), in quanto l'altro è apparso secondario rispetto all'obiettivo della presente ricerca.

Per completare l'analisi della spesa farmaceutica sono state calcolate anche alcune misure di variabilità, cioè della maggiore o minore dispersione dei 
dati attorno alle suddette medie: la deviazione standard e il range (indicando, per questo, i due valori, rispettivamente massimo e minimo).

\section{I ricoveri ospedalieri}

In una valutazione economica condotta dal punto di vista della società, la figura di costo corretta da assumere per i costi di ricovero ospedaliero è quella del costo reale della degenza. In questo studio, peraltro, data la notevole difficoltà di ricostruire tale costo, è stata adottata come proxy dello stesso la tariffa locale (cioè vigente in Sicilia) di pagamento prospettico (DRG). L'attuale classificazione del sistema di pagamento non contempla però specificamente la voce $\bullet$ morbillo , che è incluso (sotto i codici ICD-9 05579, 0558 e 0559) in un DRG di contenuto assai ampio: il 422 (malattie di origine virale e febbre di origine sconosciuta, età < 18) nonché nel 421, analogo, ma per età > 17. Il DGR 422 è stato infatti quello maggiormente utilizzato all'Ospedale G. Di Cristina su un campione di 390 casi di ricovero in seguito a morbillo nel corso dell'epidemia. Come si può osservare nella tabella III, il 93\% dei ricoveri (per morbillo tout court) era stato classificato con il DRG 422; il 6\% (per complicanze, tipicamente pneumopatie) con il DRG 091; infine, 1'1\% (encefalite) con il DRG 020.

Ponderando le rispettive tariffe di questi DRG con le frequenze suddette, si è ottenuta la tariffa media ponderata che è stata poi utilizzata per valorizzare i ricoveri ospedalieri.

\section{Le complicanze e i fattori di rischio}

Lo studio ha rilevato la presenza di fattori di rischio e di complicanze tra i pazienti. In particolare, i fattori di rischio fattori, cioè, capaci di aggravare il decorso della malattia sono risultati complessivamente presenti nel 10,2\% dei casi; le frequenze relative dei diversi fattori, previo il loro raggruppamento in alcune grandi classi di aggregazione, sono riportate nella tabella IV.

La presenza di fattori di rischio, peraltro, non è di per sé generatrice di costi a carico della malattia in corso (il morbillo, nella fattispecie). I costi sono invece associati alle complicanze cioè ad ulteriori eventi patologici collegati a quello in corso le quali sono state rilevate dallo studio in misura del $16,2 \%$ dei casi, con la distribuzione di frequenza riportata nella tabella V.

I numerosi casi di complicanze che si sono verificati sono stati in generale trattati in privato dal pediatra curante oppure nel corso di un ricovero ospedaliero. In entrambe le eventualità non si sono posti particolari problemi di calcolo dei costi: nella prima, infatti, le relative risorse sanitarie erano state rilevate nel questionario indistintamente da quelle di trattamento del morbillo quo talis e, quindi, sono poi state computate tutte insieme, mentre nella seconda il controvalore di tali risorse era già compreso nella tariffa del DRG.

L'unica eccezione a questo stato di cose è rappresentata da un caso di encefalite grave, che ha comportato una sequela di interventi terapeutici e clinici ulteriori a quelli previsti dal questionario o compresi nell'episodio di ricovero. Per questo caso, si è proceduto ad individuare tali ulteriori interventi:

day hospital: 7 accessi

fisiokinesiterapia: 2 sedute settimanali per 12 mesi logopedia: 3 sedute

RM encefalo: 1 esame

EEG: 3 esami

terapia anticonvulsivante: fenobarbitale $50 \mathrm{mg} /$ die per 18 mesi

monitoraggio (barbituremia): 3 dosaggi (1 ogni 6 mesi)

Tabella III. DRG utilizzati all Ospedale G. Di Cristina (Palermo) su un campione di 390 casi di ricovero in seguito a morbillo

\begin{tabular}{llll}
\hline DRG & & Frequenza nel campione & Tariffa locale (Lit.) \\
\hline $\mathbf{4 2 2}$ & Malattie di origine virale e febbre di origine sconosciuta, età $<18$ & $93 \%$ & 3.359 .000 \\
$\mathbf{0 9 1}$ & Polmonite semplice e pleurite, età $<18$ & $6 \%$ & 3.516 .000 \\
$\mathbf{0 2 0}$ & Infezioni del sistema nervoso, eccetto meningite virale & $1 \%$ & 8.800 .000 \\
\hline
\end{tabular}

Fonte: Comunicazione personale, Università di Palermo 
Tabella IV. Epidemia di morbillo a Palermo nel 1996-97: frequenza relativa dei fattori di rischio

\begin{tabular}{lr}
\hline Allergie & $67,0 \%$ \\
Vie respiratorie & $15,8 \%$ \\
Sintomi generali & $6,9 \%$ \\
O.R.L. & $3,4 \%$ \\
S.N.C. & $2,5 \%$ \\
Apparato gastrointestinale & $2,0 \%$ \\
Sistema cardiovascolare & $1,5 \%$ \\
Diabete & $1,0 \%$ \\
Altri fattori & $1,5 \%$ \\
\hline
\end{tabular}

assistenza continua da parte della madre: 6 mesi.

La terapia anticonvulsivante è stata valorizzata semplicemente al prezzo al pubblico del farmaco (Gardenale), senza computare altri costi annessi. Per tutte le altre risorse sanitarie, sono state utilizzate le tariffe usuali, come proxy dei rispettivi costi reali; in particolare, per il day hospital è stata utilizzata la tariffa (DRG) giornaliera per le malattie infettive e parassitarie in vigore a Palermo: Lit. 280.000.

Infine, per valorizzare l'assistenza continua da parte della madre si sono adottati i seguenti criteri. Di fatto, la madre del paziente con encefalite era lavoratrice, costretta quindi ad assentarsi dal lavoro per prestare assistenza al figlio malato e il valore stimato di una sua giornata di lavoro perduta è di Lit. 166.000 circa (si veda, per la metodologia sottostante a questa stima, il prossimo paragrafo sui costi indiretti). Tuttavia, per tenere conto del fatto che, nel campione, solo il 19,1\% delle madri si trovava nella suddetta condizione (non volendo, in altre parole, ipotizzare che ogni paziente pediatrico colpito da morbillo con encefalite debba necessariamente avere la madre lavoratrice), quell'importo è stato moltiplicato per un coefficiente probabi-

Tabella V. Epidemia di morbillo a Palermo nel 1996-97: frequenza relativa delle complicanze

\begin{tabular}{lr}
\hline Vie respiratorie & $60,1 \%$ \\
O.R.L. & $29,9 \%$ \\
Apparato gastrointestinale & $7,9 \%$ \\
S.N.C. & $1,3 \%$ \\
Sintomi generali & $0,6 \%$ \\
Sistema cardiovascolare & $0,3 \%$ \\
Altre complicanze & $7,9 \%$ \\
\hline
\end{tabular}

listico pari a 0,191 . Infine, il totale delle giornate lavorative perdute nell' arco di 6 mesi è stato calcolato sulla base di una media di 22 giornate lavorative per mese.

\section{Le visite pediatriche}

Anche le visite compiute dai pazienti presso i loro pediatri convenzionati sono state rilevate con le schede-questionario.

La loro inclusione o meno tra $\mathrm{i}$ costi diretti resta un argomento controverso in quanto, dato il sistema a capitazione attualmente vigente in Italia, la spesa marginale per il SSN di una visita è pari a zero. Di conseguenza, quantomeno negli studi di valutazione completa (tipo costo-efficacia), in cui si cerca di determinare solo le risorse aggiuntive consumate da ciascun intervento sotto esame, tale voce (in regime di quota capitaria, beninteso) potrebbe non essere considerata.

Ma nel presente lavoro, il cui obiettivo è la quantificazione di un costo sociale quale quello del morbillo in età pediatrica, si è ritenuto opportuno includere le visite perché esse sono indicative di un utilizzo (rivelatosi massiccio) di risorse sanitarie che, per quanto già messe a disposizione della collettività, hanno pur sempre nel complesso un loro costo in quanto il pediatra riceve comunque un compenso annuo.

In conclusione, a una singola visita è stato attribuito un valore monetario di Lit. 35.900, ottenuto dividendo la quota capitaria annua (Lit. 158.000) per una stima del numero medio di visite per assistito all'anno $(4,4)$, basata su dati di recente pubblicazione. ${ }^{[20,21]}$

\section{I costi indiretti (valore economico del tempo perso a causa della malattia)}

Come si era anticipato, sulla base delle informazioni raccolte con i questionari si è cercato di stimare anche due voci di costi indiretti: una, che si potrebbe dire classica in un genere di analisi come questa, è quella imputabile all'assenza dal lavoro nella fattispecie l'assenza lavorativa della madre del paziente per poter essere disponibile ad accudire quest'ultimo; la seconda è quella legata all'assenza scolastica del paziente nel corso della malattia. 


\section{L'assenza lavorativa della madre del paziente}

Nel calcolo dei costi indiretti per assenza dal lavoro, generalmente viene attribuito a ogni giorno di assenza un valore corrispondente a quello della produzione mancata a causa dell'assenza stessa valore che, secondo la teoria cosiddetta del capitale umano, si assume nella pratica equivalente al costo totale di lavoro per lo stesso periodo. ${ }^{[22]}$ L'ipotesi implicita è dunque che il lavoratore assente non abbia potuto essere sostituito, donde il calo nella produzione. Ma anche se invece, per ipotesi, egli fosse stato sostituito, rimanendo così la produzione costante, si dovrebbe in questo caso tenere conto del conseguente costo di lavoro aggiuntivo (il lavoratore assente continua infatti a pesare economicamente attraverso il sistema assicurativo); è evidente in tale ragionamento l'ipotesi che la sostituzione sia avvenuta con ulteriore forza lavoro, e non

invece recuperando margini di produttività disponibili tra gli attuali occupati.

In ogni caso, la valorizzazione del costo indiretto in questione è stata qui effettuata sulla base del costo complessivo (ovvero del reddito) di lavoro per il tempo corrispondente. Si è posto innanzitutto il problema di riportare il dato disponibile a livello naziona$\mathrm{l}^{[23]}$ del reddito medio annuo pro capite da lavoro dipendente al livello economico locale; per far questo, sulla base dei dati regionali del PIL, ${ }^{[24]}$ si è costruito un numero indice di quello siciliano, considerando 100 la media nazionale; tale indice è poi stato aumentato di un $10 \%$ per tenere in qualche modo conto della realtà urbana e, quindi, della maggior valenza economica di Palermo rispetto alla media dell'intera isola. Il dato di reddito annuo $\bullet$ localizzato con tale indice è stato infine diviso per un numero convenzionalmente accettabile di 220 giornate lavorative annue; così, ogni giorno di assenza dal lavoro da parte della madre di un paziente ha comportato un costo indiretto di circa Lit. 166.000.

Non è stato possibile raccogliere con il questionario informazioni sulla durata di tale assenza espressa in giorni. Essa è stata stimata da un esperto in 6 giorni lavorativi.

\section{L'assenza scolastica del paziente}

Si è ritenuto opportuno attribuire, sia pure a titolo più che altro simbolico, un peso economico al- l'assenza scolastica dei pazienti in età della scuola dell'obbligo (dai 6 ai 14 anni). Dei 2.031 pazienti censiti nel database, 872 rientravano in tale età. Per la precisione, questa era l'età al momento dell'insorgenza del morbillo; di conseguenza, un ipotetico paziente che avesse avuto sei anni e due mesi quando, ad esempio, nel marzo del 1997 contraeva la malattia, in realtà non si sarebbe ancora trovato in regime scolare (per iscriversi alla prima elementare è necessario che si compiano sei anni entro l'anno di iscrizione). Escludendo dunque i casi impropri, quelli effettivamente scolarizzati sono risultati 850 . Ciò premesso, l'attribuzione di un peso economico all' assenza da scuola è apparsa motivabile sotto due aspetti: il primo è che tale assenza, privando pro tempore i pazienti degli input scolastici di socializzazione, formazione, informazione, ecc., ne riduce in proporzione il processo di crescita come futuri soggetti economici attivi, con un conseguente costo atteso per il sistema economico-sociale; il secondo è che, a fronte dell' assenza, c'è una quota del servizio educativo offerto dalla struttura scolastica che rimane una risorsa inutilizzata, e quindi costituisce un puro costo senza rientro.

Nel tentativo di valutare più che altro questo secondo aspetto del valore economico del tempo perso a causa della malattia (in quanto meno aleatorio e più definibile in termini di calcolo che non il primo), tutte le componenti (locali, attrezzature, sussidi, diversi profili di personale) della risorsa scolastica non utilizzata per via dell' assenza individuale sono state ridotte a una quota della produzione didattica dell'insegnante presente in classe. In concreto, il reddito medio annuo pro capite da lavoro dipendente nella Pubblica Amministrazione, ${ }^{[23]}$ adottato come proxy del valore di tale produzione in capo a un anno, è stato suddiviso su 205 giorni numero minimo di durata di un anno scolastico e poi su 20 alunni (cioè la media tra un minimo di 15 e un massimo di 25 per classe). In definitiva, per ogni giorno di assenza da scuola di un paziente è stato valutato un costo-opportunità di circa Lit. 15.000. Con questo termine costo-opportunità si intende qui indicare il ritorno economico che si sarebbe conseguito impiegando alternativamente e in modo altrettanto conveniente gli investimenti fatti 
Tabella VI. Spesa farmaceutica per categorie di farmaci

\begin{tabular}{|c|c|c|c|c|c|}
\hline & & $\begin{array}{l}\text { Antipiretici } \\
\text { e FANS }\end{array}$ & $\begin{array}{l}\text { Antibiotici } \\
\text { orali }\end{array}$ & $\begin{array}{l}\text { Antibiotici } \\
\text { iniettabili }\end{array}$ & $\begin{array}{l}\text { Altri } \\
\text { farmaci }\end{array}$ \\
\hline Costo medio & Lit. & 9.059 & 6.708 & 4.419 & 907 \\
\hline DS & Lit. & 5.843 & 12.995 & 30.195 & 4.283 \\
\hline Valore massimo & Lit. & 44.800 & 74.000 & 484.400 & 42.600 \\
\hline Valore minimo & Lit. & 0 & 0 & 0 & 0 \\
\hline $\begin{array}{l}\text { Costo dell epidemia } \\
\text { (per } 9.058 \text { casi) }\end{array}$ & miolit & 82,1 & 60,8 & 40,0 & 8,2 \\
\hline
\end{tabular}

Fonte: Rilevazione campionaria elaborata da Economia Sanitaria (ES)

nelle strutture scolastiche in questione.

Anche la durata dell'assenza da scuola è stata stimata da un esperto, che ha indicato in 10 giorni un possibile valore medio di tale durata.

\section{Risultati}

Innanzitutto, un dato epidemiologico di notevole interesse è emerso dalla ricerca: tenendo presente che i 2.029 casi di morbillo in età pediatrica rilevati corrispondono a una popolazione di 31.565 assistiti, si deduce che il tasso di morbilità campionario dell'epidemia è stato complessivamente del 6,4\%. Si può inoltre notare che, poiché la casistica rilevata si riferisce proprio all'arco di dodici mesi (dall'inizio di settembre 1996 a fine agosto 1997), quel tasso ha la stessa dimensione epidemiologica di un tasso annuo di incidenza.

Per quanto riguarda i risultati in termini di dati economici, questi verranno di seguito esposti nella stessa sequenza con cui erano stati presentati nel precedente paragrafo metodologico.

\section{I costi diretti}

\section{La spesa farmaceutica}

La tabella VI presenta l'analisi della spesa farmaceutica in regime ambulatoriale secondo le linee metodologiche prospettate nel precedente paragrafo metodologico.

La tabella è ripartita nelle quattro grandi categorie di farmaci in cui risultano segmentabili i dati campionari in materia: antipiretici e FANS, antibiotici orali, antibiotici iniettabili, altri farmaci.

All'interno di ciascuna ripartizione sono riportate delle statistiche (in particolare, il costo medio per il singolo caso di morbillo). Si può così osservare, ad esempio, che per un singolo caso di morbillo il costo medio in antipiretici e FANS è stato di Lit. 9.059.

Infine, per ciascuna ripartizione, risulta quantificato il rispettivo costo sulla scala epidemica di tutta la popolazione pediatrica residente a Palermo (9.058 casi, come si era detto in precedenza).

Riassumendo, e sempre facendo riferimento ai dati della tabella, la componente più forte della spesa farmaceutica nella congiuntura in oggetto è stata costituita dagli antipiretici e FANS: ovvero 82,1 milioni di lire. Su un piano intermedio si sono poste le due categorie degli antibiotici orali e iniettabili mentre un rilievo solo marginale hanno avuto gli altri farmaci.

A conclusione dell' analisi della spesa farmaceutica per categorie, la tabella VII offre un riepilogo d'insieme. Nel complesso, la spesa farmaceutica a fronte dell'epidemia di morbillo nella popolazione pediatrica residente a Palermo è valutabile in 191 milioni di lire.

A completamento della documentazione sull'argomento, nella tabella VIII viene analizzata la composizione percentuale dei consumi farmaceutici (corrispondenti alla spesa di cui si è trattato appena sopra), segmentata per principi attivi.

Come la precedente tabella di analisi, anche questa è ripartita nelle quattro categorie di farmaci utilizzati. All'interno di ciascuna categoria vengono elencati i principi attivi che sono stati prescritti dai pediatri curanti, affiancati dalla rispettiva incidenza percentuale sul totale della spesa nonché sul totale del numero delle terapie prescritte della categoria. I quattro elenchi sono ordinati secondo l'importanza dell'incidenza sulla spesa; si può peraltro 
Tabella VII. Riepilogo della spesa farmaceutica

\begin{tabular}{llr}
\hline Costo medio & Lit. & $\mathbf{2 1 . 0 9 3}$ \\
DS & Lit. & 34.008 \\
Valore massimo & Lit. & 499.200 \\
Valore minimo & Lit. & 0 \\
\hline Costo dell epidemia & miolit & $\mathbf{1 9 1 , 1}$ \\
(per 9.058 casi) & & \\
\hline Fonte: Rilevazione campionaria elaborata da ES \\
\hline
\end{tabular}

notare che esiste una discreta correlazione tra questa incidenza e quella sul numero delle terapie prescritte sia pure con alcune vistose eccezioni; ad esempio, tra gli antibiotici orali, l' amoxicillina conta per il 13,2\% della spesa complessiva, ma è stata di gran lunga la più frequentemente prescritta: più di un terzo $(35 \%)$ delle terapie di tutta la categoria, mentre, al contrario, il cefacloro ha avuto un peso relativo nelle prescrizioni $(13,9 \%)$ che è solo circa la metà di quello nella spesa $(22,2 \%)$.

Da notare, infine, che le terapie di antipiretici e FANS sono state pressoché monopolizzate (quasi al $90 \%$ ) dal paracetamolo, mentre il solo ceftriaxone è stato utilizzato in misura superiore rispetto a tutti gli altri antibiotici iniettabili nel loro insieme $(62,7 \%$ sul totale della spesa e $57,7 \%$ sul totale delle terapie prescritte nella sua categoria).

\section{I ricoveri ospedalieri}

Le 71 ospedalizzazioni campionarie $(3,5 \%$ dei pazienti colpiti) sono avvenute principalmente in seguito alle complicanze indotte dal morbillo: la presenza di casi con complicanze tra i ricoverati è stata dell' $87,3 \%$ vs il $16,2 \%$ di tutto il campione.

Valorizzando i ricoveri con la tariffa media ponderata per caso di Lit. 3.423.000 (di cui al precedente paragrafo metodologico), e sempre applicando come fattore di proiezione il reciproco della quota campionaria, risulta che i ricoveri ospedalieri sono costati in tutto 1 miliardo e 85 milioni di lire.

\section{I costi dell'encefalite}

Il caso campionario di encefalite ha comportato dei costi aggiuntivi cioè successivi a quelli del ricovero ospedaliero pari a circa 8,6 milioni di lire, come documentato dalla tabella IX (le cui assunzioni sono state commentate nel precedente paragrafo metodologico). Come si può notare, l'am- montare dei costi è determinato soprattutto dal costo indiretto (stimato) delle giornate lavorative perse dalla madre del paziente per poterlo assistere.

Il suddetto costo è stato proiettato a 4 casi attesi in tutta la popolazione pediatrica residente a Palermo; per questa operazione è possibile trovare un riscontro anche nel lavoro di Romano et al., ${ }^{[19]}$ nel quale vengono riportati 4 casi di encefalite (di cui 2 gravi) su 558 casi pediatrici di morbillo ricoverati presso l'Ospedale G. Di Cristina tra il settembre 1996 e il giugno 1997.

Per tenere conto del fatto che sui 4 casi di encefalite 2 non si erano rivelati gravi (quindi relativamente meno costosi in termini di risorse sanitarie assorbite), si è avanzata l'ipotesi che un caso non grave assorbisse metà delle risorse di uno grave; su questa base, il dato di costo proiettato a 4 casi è stato allora ridimensionato a tre quarti del valore. In definitiva, il costo della complicanza in oggetto nella popolazione pediatrica di Palermo è risultato di 26 milioni di lire.

\section{Le visite pediatriche}

Sono state rilevate nel campione 2.068 visite successive alle prime 2.029 (quelle, cioè, a inizio della malattia). Estrapolando si ha un totale di 18.290 visite che, valorizzate come sopra premesso, comportano un costo complessivo di 657 milioni di lire.

\section{I costi indiretti (valore economico \\ del tempo perso a causa della malattia)}

\section{L'assenza lavorativa della madre del paziente}

Dalla rilevazione campionaria è emerso che tra le madri dei 2.029 pazienti, 376 erano lavoratrici $(19,1 \%)$. Valorizzando a un costo economico giornaliero di 166.000 lire le 6 giornate lavorative perdute da ciascuna di loro per assistere il figlio malato (per le ipotesi relative a questi dati si veda il precedente paragrafo metodologico), si è ottenuto un costo complessivo per tutta l'epidemia di 1 miliardo e 672 milioni di lire.

\section{L'assenza scolastica del paziente}

Sulla base delle considerazioni svolte in precedenza (850 pazienti campionari in età scolare assenti da scuola per malattia per 10 giorni ciascuno, ad un costo-opportunità giornaliero di 15.000 lire), 
Tabella VIII. Composizione dei consumi farmaceutici

\begin{tabular}{|c|c|c|c|c|c|}
\hline & \multicolumn{2}{|c|}{ Antipiretici e FANS } & \multicolumn{3}{|c|}{ Antibiotici iniettabili } \\
\hline & \multicolumn{2}{|c|}{ Composizione dei consumi } & & \multicolumn{2}{|c|}{ Composizione dei consumi } \\
\hline & $\begin{array}{c}\text { sul totale } \\
\text { della spesa }\end{array}$ & $\begin{array}{c}\text { sul totale di } \\
\text { terapie prescritte }\end{array}$ & & $\begin{array}{c}\text { sul totale } \\
\text { della spesa }\end{array}$ & $\begin{array}{c}\text { sul totale di } \\
\text { terapie prescritte }\end{array}$ \\
\hline \multirow[t]{4}{*}{$\underline{\text { metamizolo }}$} & $\begin{array}{r}88,5 \% \\
6,4 \% \\
4,2 \% \\
0,9 \% \\
0,1 \% \\
100,0 \%\end{array}$ & $\begin{array}{r}89,0 \% \\
6,5 \% \\
3,1 \% \\
1,4 \% \\
0,1 \% \\
100,0 \%\end{array}$ & $\begin{array}{l}\text { ceftriaxone } \\
\text { ceftazidime } \\
\text { cefotaxime } \\
\text { cefonicid } \\
\text { cefazolina } \\
\text { cefuroxime } \\
\text { gentamicina }\end{array}$ & $\begin{array}{r}62,7 \% \\
27,3 \% \\
5,6 \% \\
2,3 \% \\
1,3 \% \\
0,5 \% \\
0,4 \% \\
100,0 \%\end{array}$ & $\begin{array}{r}57,7 \% \\
25,0 \% \\
9,6 \% \\
1,9 \% \\
1,9 \% \\
1,9 \% \\
1,9 \% \\
100,0 \%\end{array}$ \\
\hline & \multicolumn{2}{|c|}{ Antibiotici orali } & & \multicolumn{2}{|c|}{ Altri farmaci } \\
\hline & \multicolumn{2}{|c|}{ Composizione dei consumi } & & \multicolumn{2}{|c|}{ Composizione dei consumi } \\
\hline & $\begin{array}{c}\text { sul totale } \\
\text { della spesa }\end{array}$ & $\begin{array}{c}\text { sul totale di } \\
\text { terapie prescritte }\end{array}$ & & $\begin{array}{c}\text { sul totale } \\
\text { della spesa }\end{array}$ & $\begin{array}{l}\text { sul totale di } \\
\text { terapie prescritte }\end{array}$ \\
\hline claritromicina & $25,5 \%$ & $18,7 \%$ & acetilcisteina & $60,3 \%$ & $30,6 \%$ \\
\hline cefacloro & $22,2 \%$ & $13,9 \%$ & beclometasone & $18,5 \%$ & $11,3 \%$ \\
\hline amoxicillina + & $20,9 \%$ & $16,3 \%$ & salbutamolo & $8,8 \%$ & $28,1 \%$ \\
\hline acido clavulanico & & & carbocisteina & $3,9 \%$ & $5,6 \%$ \\
\hline amoxicillina & $13,2 \%$ & $35,0 \%$ & betametasone & $1,6 \%$ & $6,9 \%$ \\
\hline cefetamet & $5,59 \%$ & $4,4 \%$ & diazepam & $1,2 \%$ & $2,5 \%$ \\
\hline ceftibuten & $4,9 \%$ & $3,5 \%$ & vitamina $B$ & $1,1 \%$ & $1,3 \%$ \\
\hline cefixima & $3,7 \%$ & $4,0 \%$ & ambroxolo & $1,0 \%$ & $1,9 \%$ \\
\hline rokitamicina & $1,6 \%$ & $1,2 \%$ & levodropropizina & $0,9 \%$ & $1,3 \%$ \\
\hline eritromicina & $1,25 \%$ & $2,0 \%$ & vitamina C & $0,8 \%$ & $3,1 \%$ \\
\hline azitromicina & $0,8 \%$ & $0,8 \%$ & domperidone & $0,6 \%$ & $4,4 \%$ \\
\hline josamicina & $0,1 \%$ & $0,2 \%$ & ipratropio bromuro & $0,4 \%$ & $0,6 \%$ \\
\hline & $100,0 \%$ & $100,0 \%$ & $\begin{array}{l}\text { metoclopramide } \\
\text { ketotifene } \\
\text { cloperastina } \\
\text { vitamine }\end{array}$ & $\begin{array}{r}0,3 \% \\
0,3 \% \\
0,2 \% \\
0,1 \% \\
100,0 \%\end{array}$ & $\begin{array}{r}0,6 \% \\
0,6 \% \\
0,6 \% \\
0,6 \% \\
100,0 \%\end{array}$ \\
\hline
\end{tabular}

Fonte: Rilevazione campionaria elaborata da ES

il costo complessivo delle assenze scolastiche è risultato di 569 milioni di lire.

\section{Il costo complessivo dell'epidemia}

La tabella $\mathrm{X}$, oltre a riassumere le grandi componenti di costo (e i rispettivi valori) considerate nella presente ricerca, mostra come l'epidemia di morbillo del 1996-97 nella città di Palermo sia costata complessivamente, per quanto riguarda la popolazione pediatrica, 4,2 miliardi di lire, in cui i costi diretti (cioè i meno questionabili) corrispondevano a quasi la metà $(46,6 \%)$. La componente più signi- ficativa dei costi diretti $(55,4 \%)$ era riconducibile ai ricoveri ospedalieri, mentre i costi indiretti erano costituiti per tre quarti dalla forzata assenza dal lavoro delle madri dei pazienti.

Mediamente, un singolo caso è costato circa Lit. 464.000. In termini di soli costi diretti, l'analogo costo è stato di Lit. 217.000 circa.

\section{Risorse assorbite: un confronto \\ tra i pazienti vaccinati e quelli non vaccinati}

Dei 2.029 pazienti con morbillo censiti dalla rilevazione, 120 (il 6\% circa) erano stati in prece- 
Tabella IX. Costi aggiuntivi(a) di un caso di encefalite grave

\begin{tabular}{lcccc}
\hline Risorse & Valore unitario (Lit.) & Frequenza & Totale (Lit.) & $\%$ \\
\hline Day Hospital & $280.000^{(\mathrm{b})}$ & 7 & 1.960 .000 & 22,9 \\
Fisiokinesiterapia & $17.600^{(\mathrm{c})}$ & $96^{(\mathrm{d})}$ & 1.689 .600 & 19,8 \\
Logopedia & $16.300^{(\mathrm{c})}$ & 3 & 48.900 & 0,6 \\
RM & $430.000^{(\mathrm{c})}$ & 1 & 430.000 & 5,0 \\
EEG & $45.000^{(\mathrm{c})}$ & 3 & 135.000 & 1,6 \\
Fenobarbitale & $103,3^{(\mathrm{e})}$ & $540^{(\mathrm{g})}$ & 55.800 & 0,7 \\
Monitoraggio & $15.500^{(\mathrm{c})}$ & 3 & 46.500 & 0,5 \\
Assistenza materna & $31.706^{(\mathrm{g})}$ & $132^{(\mathrm{h})}$ & 4.185 .192 & 48,9 \\
\hline Totale & & & $\mathbf{8 . 5 5 0 . 9 9 2}$ & $\mathbf{1 0 0 , 0}$ \\
\hline
\end{tabular}

(a) Costi di follow-up, successivi al ricovero ospedaliero.

(b) DRG.

(c) Tariffa ambulatoriale.

(d) 2 sedute settimanali per 12 mesi.

(e) Costo (prezzo al pubblico) di una cpr da $50 \mathrm{mg}$.

(f) $50 \mathrm{mg} /$ die per 18 mesi.

(g) Reddito/die, condizionato probabilisticamente.

(h) 6 mesi, di 22 giorni lavorativi ciascuno.

Fonte: Dati sulle risorse aggiuntive comunicati dall Università di Palermo, tariffe ministeriali[ ${ }^{[25]}$ e dati ISTAT $^{[23]}$

denza vaccinati, pur senza conseguirne una totale protezione. È parso interessante tentare un confronto tra questo sottoinsieme e quello dei non vaccinati per vedere se la condizione di trovarsi vaccinati anche senza una totale protezione al momento dell'insorgere della malattia facesse qualche differenza sull'impatto di quest'ultima in termini di risorse consumate e relativi costi.

La tabella XI riporta i risultati indicativi di un primo confronto sotto il profilo clinico ed epidemiologico, da cui risultano alcune differenze statisticamente significative.

Nella prima delle tre fasce in cui è suddivisa la tabella si rileva che i pazienti vaccinati (la vaccinazione era avvenuta, nella massima parte dei casi, all'età di 1 anno e 6 mesi) hanno contratto il morbillo a un'età leggermente inferiore (4 anni e 11 mesi) rispetto agli altri (5 anni e 7 mesi). Ben il 54,2\% dei vaccinati aveva la madre lavoratrice, contro il $16,3 \%$ del gruppo di confronto $(\mathrm{p}<0,01)$ : sembra dunque che lo status occupazionale (e, in questo senso, sociale) della madre influisca positivamente sull'opzione in merito alla prevenzione vaccinale del figlio.

La seconda fascia della tabella è dedicata al confronto tra i consumi di terapie farmacologiche. Questi vengono misurati in due modi (tali da renderli, appunto, confrontabili): come percentuale dei casi trattati rispetto al totale (trattato e non) e come durata media della terapia.

Anche in questo caso si ha la ripartizione delle terapie nelle quattro categorie di farmaci già più volte incontrata. Così, si può notare che il consumo di antipiretici e FANS è stato leggermente inferiore tra $\mathrm{i}$ vaccinati, sia in termini di casi trattati $(75,8 \%$ vs $80,6 \%$; $\mathrm{p}<0,2$ ) che di durata della terapia ( 3 vs 3,7 giorni; $\mathrm{p}<$ $0,01)$. Ma la più evidente differenza emerge nel consumo di antibiotici orali: $14,2 \%$ di casi trattati nei vaccinati vs 30,3\% nei non vaccinati $(\mathrm{p}<0,01)$. L'altra differenza di rilievo consiste nel fatto che tra i vaccinati non c'è stato alcun ricorso né agli antibiotici iniettabili $(\mathrm{p}<0,1)$ né agli altri farmaci $(\mathrm{p}<0,01)$.

Nella terza fascia della tabella si nota che il tasso di complicanze tra i vaccinati $(8,3 \%)$ è stato circa la metà di quello degli altri $(17,5 \%$; $p<0,01)$; peraltro, tra i vaccinati erano presenti in misura minore sia i fattori di rischio delle stesse complicanze $(5,8 \%$ vs $10,4 \%)$ che le patologie concomitanti $(0,8 \%$ vs $2,1 \%)$.

Il tasso di ospedalizzazione tra i vaccinati è stato dello $0,8 \%$ vs il $3,7 \%$ dei non vaccinati $(\mathrm{p}<0,1)$.

In entrambi i gruppi si è infine registrato lo stesso numero di visite pediatriche ( 2 in media per paziente).

Un secondo confronto tra il sottoinsieme dei pa- 
Tabella X. Epidemia di morbillo a Palermo nel 1996-97: costo complessivo

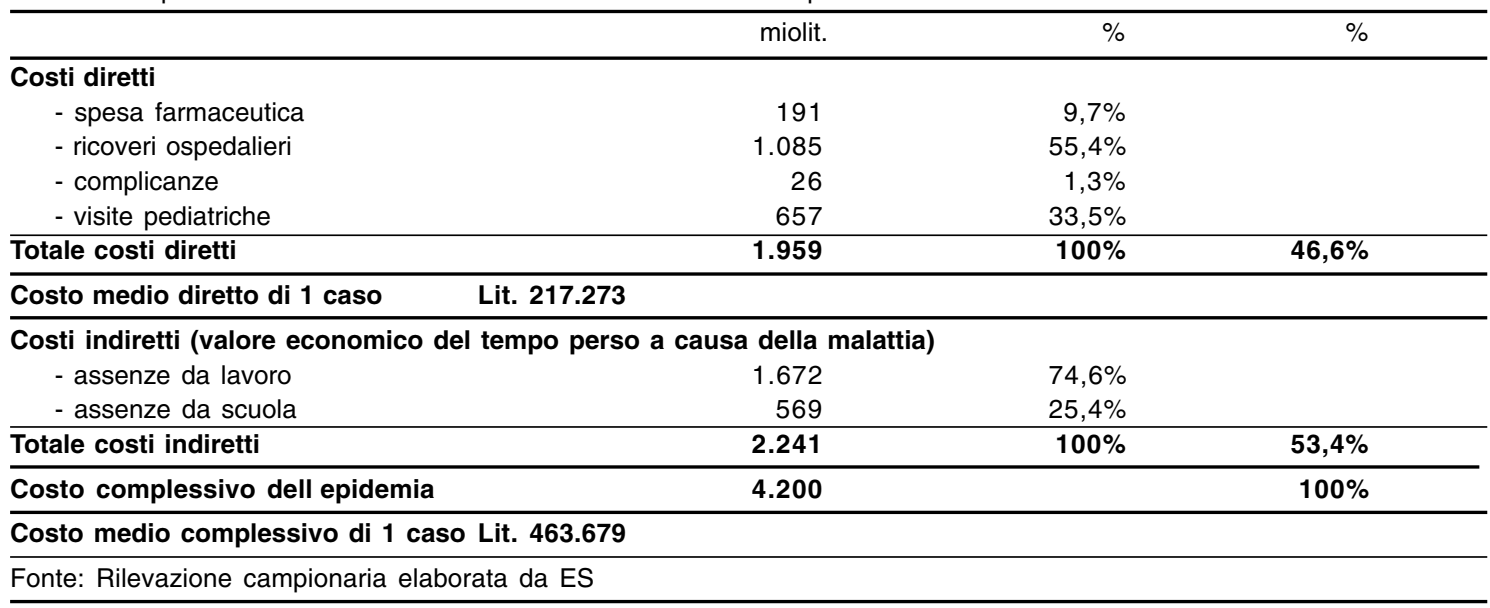

zienti vaccinati e quello dei non vaccinati è stato realizzato sotto il profilo dei costi, ed è rappresentato nella tabella XII in termini di costi medi (si precisa che sono i costi medi rapportati alla totalità dei casi e non ai soli utilizzatori delle rispettive risorse).

La tabella XII conferma, in termini di costi, le informazioni illustrate nella tabella precedente in termini di risorse fisiche. In particolare, si può notare che il costo diretto medio nei pazienti vaccinati (Lit. 110.346) non raggiunge la metà dell'analogo nel gruppo di confronto (Lit. 223.373), grazie al minor ricorso alle terapie farmacologiche e ai ricoveri ospedalieri verificatosi tra i primi, che si è tradotto in rispettivi minori costi medi.

\section{Discussione}

Vengono di seguito ripresi e commentati alcuni punti $\bullet$ critici contenuti nel corso del presente studio o comunque meritevoli di rilievo.

\section{Un tentativo di stima in prima approssimazione della percentuale di sottonotifica locale}

Le notifiche di morbillo in età pediatrica a Palermo e provincia nel 1997 sono state 3.341, secondo il dato comunicato dalla Regione Sicilia al Ministero della Sanità.

Tabella XI. Confronto tra pazienti vaccinati e non vaccinati

\begin{tabular}{|c|c|c|c|c|c|c|c|c|}
\hline Dati generali & \multicolumn{2}{|l|}{ N. casi } & $\begin{array}{l}\text { Età media } \\
\text { (anni) }\end{array}$ & \multicolumn{2}{|c|}{$\begin{array}{l}\text { Età media alla } \\
\text { vaccinazione (anni) }\end{array}$} & \multicolumn{3}{|c|}{$\begin{array}{l}\text { Madre lavoratrice } \\
(\% \text { sui casi) }\end{array}$} \\
\hline Vaccinati & \multicolumn{2}{|l|}{120} & 4,9 & \multicolumn{2}{|l|}{1,5} & \multicolumn{3}{|l|}{$54,2 \%$} \\
\hline Non vaccinati & \multicolumn{2}{|l|}{1.909} & \multicolumn{3}{|c|}{5,7} & \multicolumn{3}{|l|}{$16,3 \%$} \\
\hline \multirow[t]{2}{*}{ Terapie } & \multicolumn{2}{|c|}{ Antipiretici/FANS } & \multicolumn{2}{|c|}{ Antibiotici orali } & \multicolumn{2}{|c|}{ Antibiotici iniettabili } & \multicolumn{2}{|l|}{ Altri farmaci } \\
\hline & (\% sui casi) & $\overline{(g g \text { terapia) }}$ & (\% sui casi) & $\overline{\text { (gg. terapia) }}$ & (\% sui casi) & $\overline{\text { (gg terapia) }}$ & (\% sui casi) & $\overline{\text { (gg terapia) }}$ \\
\hline Vaccinati & $75,8 \%$ & 3,0 & $14,2 \%$ & 6,2 & - & - & - & - \\
\hline Non vaccinati & $80,6 \%$ & 3,7 & $30,3 \%$ & $6,02,7 \%$ & 6,2 & $8,4 \%$ & 4,7 & \\
\hline $\begin{array}{l}\text { Complicanze } \\
\text { e ricoveri }\end{array}$ & \multicolumn{2}{|c|}{$\begin{array}{l}\text { Complicanze } \\
\text { (\% sui casi) }\end{array}$} & $\begin{array}{l}\text { Fattori di rischio } \\
\text { (\% sui casi) }\end{array}$ & \multicolumn{2}{|c|}{$\begin{array}{l}\text { Patologie concomitanti } \\
\text { (\% sui casi) }\end{array}$} & \multicolumn{2}{|c|}{$\begin{array}{l}\text { Ricoveri ospedalieri } \\
\text { (\% sui casi) }\end{array}$} & $\begin{array}{l}\text { N. medio } \\
\text { visite }\end{array}$ \\
\hline$\overline{\text { Vaccinati }}$ & \multicolumn{2}{|c|}{$8,3 \%$} & $5,8 \%$ & \multicolumn{2}{|c|}{$0,8 \%$} & \multicolumn{2}{|l|}{$0,8 \%$} & 1,97 \\
\hline Non vaccinati & \multicolumn{2}{|l|}{$17,5 \%$} & $10,4 \%$ & \multicolumn{2}{|l|}{$2,1 \%$} & \multicolumn{2}{|l|}{$3,7 \%$} & 2,03 \\
\hline
\end{tabular}

Fonte: Rilevazione campionaria elaborata da ES 
Tabella XII. Costi nel gruppo dei pazienti vaccinati e in quello dei non vaccinati

\begin{tabular}{lcc}
\hline Categorie di costo & \multicolumn{2}{l}{$\begin{array}{l}\text { Costo medio per paziente } \\
\text { (Lit.) }\end{array}$} \\
\cline { 2 - 3 } & vaccinati & non vaccinati \\
\hline Costi diretti & & \\
Farmaci & 6.878 & 9.250 \\
- antipiretici e FANS & 3.143 & 6.932 \\
- antibiotici orali & - & 4.697 \\
- antibiotici iniettabili & - & 964 \\
- altri farmaci & 10.021 & 21.843 \\
Totale & 28.525 & 125.516 \\
Ricoveri ospedalieri & - & 4.214 \\
Complicanze (encefalite) & -71.800 & 71.800 \\
Visite pediatriche & $\mathbf{1 1 0 . 3 4 6}$ & $\mathbf{2 2 3 . 3 7 3}$ \\
\hline \multicolumn{2}{c}{ Totale costi diretti } & \multicolumn{2}{c}{. } & \\
\hline Fonte: Rilevazione campionaria elaborata da ES
\end{tabular}

Volendo tentare un riscontro del dato, i passi potrebbero essere i seguenti:

come già argomentato in precedenza, dividendo i 2.029 casi di morbillo rilevati nel campione per la quota campionaria $(22,4 \%)$ si ottengono 9.058 casi di epidemia su tutta la popolazione pediatrica di Palermo città

dei 2.029 casi di morbillo, il 16\% si era manifestato nel 1996; detraendo questa percentuale dai 9.058 casi suddetti, si ottengono 7.584 casi verificatisi nel corso del 1997 fino al mese di agosto; mancherebbero, per avere il dato complessivo dell'anno, i dati dell'ultimo quadrimestre, ma si suppongono di entità trascurabile

la popolazione pediatrica di Palermo provincia è pari a 238.803 soggetti (dato reperibile sul sito Internet del Ministero della Sanità), quindi maggiore di un fattore 1,7 a quella di Palermo città (141.077 soggetti, dato comunicato dall'Università di Palermo). Ipotizzando per la provincia un tasso di morbilità pari a quello della città, e applicando detto fattore ai 7.584 casi, si avrebbero per Palermo e provincia 12.893 casi di morbillo pediatrici riferibili al 1997

poiché è lecito supporre che il tasso di morbilità in provincia sia in realtà inferiore a quello della città, la stima del numero di casi pediatrici di morbillo in Palermo e provincia nel 1997 sopra effettuata (12.893 casi) va considerata come un limite superiore. Comunque, ricordando che le notifiche sono state 3.341 , la percentuale di notifica sarebbe il $26 \%$ dei casi di malattia effettivi; in pratica, ciò significa che sarebbe stato notificato un caso di morbillo ogni quattro. In conclusione, una buona stima del fattore di sottonotifica potrebbe essere di un caso di morbillo notificato ogni tre-quattro.

\section{Critica dell'importanza dei (minori) fattori di rischio nel gruppo dei pazienti vaccinati rispetto a quello dei non vaccinati}

Va detto che il rapporto tra fattori di rischio e complicanze insorte non risulta essere così marcato. Infatti, su tutto il campione di 2.029 casi, con 202 casi in cui erano presenti fattori di rischio, si sono avuti 329 casi con complicanze dei quali solo $104(33,7 \%)$ si accompagnavano ai fattori di rischio (in altre parole, 98 casi su 202 con fattori di rischio non avevano avuto complicanze, mentre 125 casi su 329 di complicanze si erano verificati senza fattori di rischio preesistenti) e nel gruppo dei pazienti vaccinati, con 7 casi a rischio, si sono avuti 9 casi di complicanze di cui solo $1(11,1 \%)$ era anche un caso a rischio. Ipotesi di causalità, e quindi di spiegazione, tra le due variabili vanno dunque prospettate con molta prudenza.

In altre parole, parrebbe lecito pensare che non solo o non tanto la minor presenza di fattori di rischio nel gruppo dei vaccinati, ma la già avvenuta vaccinazione sia stata all'origine del minor tasso di complicanze (e quindi di ospedalizzazione nonché di consumo farmacologico) registrati nel sottoinsieme dei pazienti vaccinati rispetto a quello dei non vaccinati.

Su questo punto, rimane da osservare che lo status di $\bullet$ madre professionalmente occupata è risultato significativamente più frequente nel gruppo dei vaccinati rispetto all'altro. Ora, anche tale status (in quanto potenziale indicatore di altre variabili socio-economiche, quali migliore abitazione/alimentazione/igiene, ecc.) potrebbe aver contribuito ai differenti esiti clinici tra i due gruppi. Si tratta di un'ipotesi certamente plausibile, che meriterebbe di essere verificata, sulla base di ulteriori dati. 


\section{Considerazioni sui costi}

Premesso quanto sopra, rimane da osservare che il costo complessivo di 4,2 miliardi di lire comportato dall'epidemia di morbillo del 1996-97 nella popolazione pediatrica di Palermo città può sembrare non così rilevante in assoluto; in particolare, $i$ costi diretti ( 2 miliardi di lire) corrispondono allo $0,24 \%$ della spesa sanitaria della Regione, che nel 1996 è stata di circa 8 mila miliardi. ${ }^{[26,27]}$

Rimane comunque il fatto che si è trattato di un evento fortemente disturbante per il paziente, per la sua famiglia e per la struttura di cura perché concentrato nel tempo; se infatti si osserva la distribuzione percentuale dei casi di epidemia lungo il periodo considerato (tabella XIII) si può notare che più di tre quarti dei casi si sono verificati in soli quattro mesi (i primi del 1997).

Inoltre, si sono avute ospedalizzazioni nel 3,5\% dei casi (i costi ospedalieri sono stati la principale voce dei costi diretti).

Nella sua composizione, tutto il costo sociale dell'epidemia è risultato abbastanza bilanciato tra costi diretti e indiretti, seppure con una leggera prevalenza dei secondi. Purtroppo, non è possibile riportare qui dei riscontri di alcun genere dalla letteratura, come era del resto già stato premesso nei paragrafi iniziali.

A proposito di costi diretti, bisogna precisare che il costo di un caso di encefalite (che ne costituisce una delle voci) comprende anche una componente

Tabella XIII. Distribuzione percentuale nel tempo dei casi di morbillo (settembre 1996 - agosto 1997)

\begin{tabular}{llr}
\hline 1996 & settembre & $0,6 \%$ \\
& ottobre & $1,5 \%$ \\
& novembre & $4,6 \%$ \\
& dicembre & $9,2 \%$ \\
& gennaio & $17,6 \%$ \\
& febbraio & $23,9 \%$ \\
& marzo & $21,6 \%$ \\
& aprile & $13,9 \%$ \\
& maggio & $4,7 \%$ \\
& giugno & $1,7 \%$ \\
& luglio & $0,2 \%$ \\
& agosto & $0,2 \%$ \\
\hline
\end{tabular}

di costi indiretti (per l'assenza dal lavoro della madre), che avrebbe dovuto essere scorporata e inserita negli altri costi indiretti. Tuttavia, data l'oggettiva esiguità degli importi in questione, nonché per ragioni di semplicità espositiva, è parsa giustificata l'economia di lavoro conseguente al non aver eseguito tale scorporo.

\section{Conclusioni}

Le malattie infettive costituiscono ancora un problema anche per Paesi economicamente sviluppati.

Il morbillo fa parte del gruppo delle malattie infettive prevenibili. Tuttavia, la vaccinazione antimorbillo non è obbligatoria in Italia, dove si stima abbia raggiunto mediamente una quota del $40-45 \%$ della popolazione.

A Palermo, l'epidemia verificatasi a cavallo tra il 1996 e il 1997 ha colpito il 6,4\% della popolazione in età pediatrica (il 10,3\% di quella in età dai 0 ai 6 anni).

Scopo del presente lavoro è stato quello di valutarne il costo sociale. A tal fine è stata effettuata una rilevazione campionaria su 38 pediatri convenzionati, con un numero di assistiti (31.565) pari al 22,4\% dell'intera popolazione pediatrica di Palermo città.

L'analisi, condotta dal punto di vista della società, ha rilevato che ciascun caso di morbillo è costato quasi mezzo milione (circa Lit. 464.000). Complessivamente, l'epidemia è costata 4,2 miliardi di lire.

Di questi, poco più della metà $(53 \%)$ era dovuta ai costi indiretti, principalmente (75\%) per assenze dal lavoro delle madri lavoratrici per assistere i figli.

I costi diretti sono stati complessivamente $2 \mathrm{mi}$ liardi di lire circa, pari allo $0,24 \%$ della spesa sanitaria della Regione Sicilia.

La composizione di tali costi mostra che più della metà (il 55\%, cioè 1,1 miliardi di lire) è dovuta ai ricoveri ospedalieri (il tasso di ospedalizzazione tra i colpiti da morbillo è risultato pari al 3,5\%). L'altra importante componente di costo, che pesa per un terzo $(33 \%)$, è data dalle visite pediatriche $(657 \mathrm{mi}-$ liardi di lire).

Alla spesa farmaceutica in regime ambulatoriale (191 milioni di lire) è attribuibile un ulteriore $10 \%$ dei costi diretti. La categoria di farmaci più utilizzata, sotto il profilo dei costi complessivamente 
sostenuti, è risultata essere quella degli antipiretici e FANS (43\% della spesa complessiva), seguita dagli antibiotici orali (32\%) e da quelli iniettabili (21\%). In particolare, tra gli antipiretici il farmaco più utilizzato è stato il paracetamolo $(90 \%$ circa dei consumi a valore); tra gli antibiotici orali, tre principi attivi (claritromicina, cefacloro e amoxicillina con o senza acido clavulanico) e, tra gli iniettabili, due (ceftriaxone e ceftazidime) hanno assorbito oltre 1'80\% dei consumi.

Lo studio ha inoltre messo in evidenza che se un soggetto vaccinato contrae il morbillo, va incontro a una forma più lieve di malattia, come risulta dal minor assorbimento di risorse da parte dei vaccinati rispetto ai non vaccinati: grazie alla minor presenza di complicanze tra i vaccinati, uno solo su 120 casi ha dovuto essere ricoverato (tasso di ospedalizzazione dello $0,8 \%$, vs il $3,7 \%$ tra i non vaccinati), nessuno ha fatto ricorso a terapie antibiotiche iniettive e solo il 14\% (vs il 30,3\%) a terapie antibiotiche orali. Sotto il profilo dei costi diretti, un caso di morbillo in paziente vaccinato è costato Lit. 110.000, contro Lit. 223.000 tra i pazienti non vaccinati (cioè meno della metà).

Giova ancora ricordare che lo studio ha preso in considerazione la sola popolazione in età pediatrica residente a Palermo. Della popolazione colpita in età adulta questa ricerca non può dire nulla; il costo stimato di 4,2 miliardi di lire non può quindi essere considerato tutto il costo sociale dell'epidemia. Tuttavia, poiché a Palermo e provincia ben il $96 \%$ dei casi di morbillo (stando ai dati delle notifiche) si verifica tra la popolazione pediatrica, si può ritenere che l'ordine di grandezza di quella stima conservi una sua validità anche con riferimento all'intera popolazione.

Per concludere, infine, lo studio partendo da un problema di determinazione del costo sociale è arrivato a sviluppare gran parte delle informazioni economiche necessarie a un'eventuale analisi di costo-efficacia.

\section{Ringraziamenti}

Si ringraziano A. Romano e A. Musca per la loro preziosa collaborazione. Questa ricerca è stata resa possi- bile da un finanziamento messo a disposizione da SmithKline Beecham S.p.A.

\section{Bibliografia}

1. van den Oever R, de Graeve D, Hepp B, et al. Pharmacoeconomics of immunisation. A review. PharmacoEconomics 3 (4): 286-308, 1993

2. World Health Organization. Week Epidemiol Rec 3: 11-12, 1992

3. London WP, Yorke JA. Recurrent outbreaks of measles, chickenpox and mumps. Seasonal variations in contact rates. Am J Epidemiol 98: 453-468, 1973

4. Hirsch A. Handbuch der Historisch-geographiscen Pathologie. Enke, Stuttgart, 1881

5. Schenzle D. An age-structured model pre- and post-vaccination measles transmission. IMA Journal of Mathematics Applied in Medicine \& Biology 1: 169-191, 1984

6. Conway SP, Philips RR. Morbidity in whooping cough and measles. Archives of Disease in Childhood 64: 1442 1445, 1989

7. Centers for Disease Control. Measles prevention: recommendations of the immunization practices advisory committee. Morbidity and Mortality Weekly Report 38: 1-18, 1989

8. Centers for Disease Control. Measles. United States. Morbidity and Mortality Weekly Report 38: 601-605, 1989

9. Centers for Disease Control. Measles. United States. Morbidity and Mortality Weekly Report 40: 369-370, 1991

10. Conway SP, Leese B. Routine child immunisation. Is it worth of? PharmacoEconomics 3 (3): 183-191, 1993

11. Barry J. The misery of measles for patients and their families. Communicable Disease Report 46 (3), 1988

12. Weekly Epidemiology Records. Measles surveillance 1982-1990, Belgium 43: 314-317, 1991

13. Morbidity and Mortality Weekly Reports. Measles Prevention. Report No. 31, pp. 217-231, 1982

14. World Health Organization - Week Epidemiol Rec 19: 142-144, 1989

15. Agur Z, Cojocaru L, Mazor G, et al. Pulse mass measles vaccination across age cohorts. Proc Natl Acad Sci USA 90: 11698-11702, 1993

16. Sellik JA. The economics of screening measles. PharmacoEconomics 2 (6): 440-443, 1992

17. Mast EE, Berg JL, Hanrahan LP, et al. Risk factors for measle in a previously vaccinated population and cost-effectiveness of revaccination strategies. JAMA 264 (19): 2529-2533, 1990

18. Robertson SE, Markowitz LE, Berry DA, et al. A million dollar measles outbreak: epidemiology, risk factors, and a selective revaccination strategy. Public Health Reports 107 (1): 24-31, 1992

19. Romano A, Scarlata F, Di Carlo P, et al. Analisi descrittiva di un'epidemia di morbillo a Palermo dal settembre 1996 al giugno 1997. Insuccesso di una campagna vaccinale. Minerva Pediatrica Vol. 47: 1-7, 1998

20. Brivio L. Tre anni di pediatria di famiglia: un tentativo di autovalutazione. Medico e Bambino 17: 29-384, 1998

21. Buzzetti R, Venturelli L. Pediatria di base a Bergamo. Medico e Bambino 10: 30-33, 1988

22. Jönsson B, Stålhammer NO. The cost effectiveness of omeprazole and ranitidine in intermittent and maintenance treatment of reflux oesophagitis. The case of Sweden. British Journal of 
Medical Economics 6: 111-126, 1993

23. ISTAT. Annuario Statistico Italiano 1992, 1993, 1994, 1995, 1996, 1997

24. Il Sole-24 Ore. Elaborazione su dati ISTAT e Istituto Tagliacarne, 5 maggio 1997

25. Ministero della Sanità. Tariffe delle prestazioni di assistenza ospedaliera. Decreto Ministeriale 14 dicembre 1994, Supplemento ordinario alla Gazzetta Ufficiale n. 300 del 24 dicembre 1994

26. Ministero della Sanità. Rendiconti trimestrali delle unità sanitarie locali. $4^{\circ}$ trimestre 1996. Sistema Informativo Sanitario, situazione al 6/10/1997
27. Ministero della Sanità. Prestazioni di assistenza specialistica ambulatoriale erogabili nell'ambito del Servizio Sanitario Nazionale e relative tariffe. Decreto Ministeriale 22 luglio 1996, Supplemento ordinario alla Gazzetta Ufficiale n. 216 del 14 settembre 1996

Indirizzo per la corrispondenza: Dr. Carlo Lucioni, Istituto di Economia Sanitaria, Via Petrarca 13, 20123 Milano, Italia.

E-mail: ecsan@albert.net 